\title{
Development of ATC Tower Systems: Electronic Flight Strip System at LKPR and the Implementation of TAXI Milestone to SUM
}

\section{Pavel Černý ${ }^{1}$, Matěj Nesvadba², Stanislav Pleninger ${ }^{1 *}$}

\author{
${ }^{1}$ Department of Air Transport, Faculty of Transportation Sciences, Czech Technical University in Prague, Prague, \\ Czech Republic \\ ${ }^{2}$ Air Navigation Services of the Czech Republic, Jeneč, Czech Republic \\ *Corresponding author: Czech Technical University in Prague, Faculty of Transportation Sciences, Department of Air \\ Transport, Horská 3, 12803 Prague, Czech Republic, Email: pleninger@fd.cvut.cz
}

\begin{abstract}
This article describes the electronic flight strip system used at Václav Havel Airport Prague and presents the results of an analysis of the clearance input from air traffic control officers. The outcomes indicate that various clearances are frequently issued together in a common time frame, usually depending on the traffic flow structure. The focus then moves towards the implementation of the TAXI milestone into the Start-Up manager. The used statistic methods indicate that the amount of target take-off time recalculations after actual off-block time decreases, but not significantly. However, the accuracy of target take-off time after TAXI clearance is considerably better. The mean and most common deviation of actual take-off time from target take-off time after the implementation of TAXI milestone is -1 minute. The results are challenged and confirmed by a further analysis of the target take-off time - actual take-off time difference regarding individual stand/RWY pairs. The results may be used to adjust the assumed taxi times.
\end{abstract}

\section{Keywords}

Clearance - Electronic flight strip system — Start-Up Manager — TAXI milestone

\section{Introduction}

The Electronic Flight Strip System (EFSS) was implemented at the Václav Havel Airport Prague control tower in May 2013 [1]. There have been five main reasons that made this system beneficial:
1. Unification of working procedures

2. Integration of Advanced Safety Nets

3. Direct cooperation with SUM and A-CDM

4. Datalink connection to aircraft

5. Unified TWR workstations 
As the system was brand new with potential imperfections, there was a need to analyze the way the air traffic control officers (ATCOs) use it and possibly make appropriate adjustments. The question was, how demanding the system is towards the ATCOs and if there is any room for improvement.

An analysis of the clearance input is performed with the intention to find out how often are the individual clearances issued in peak times, whether they are issued separately or in a common timeframe, and possibly the reason for this behavior. The results may then be used to detect situations that might be overly demanding.

July 2014 has seen an enhancement of the Start-Up Manager (SUM): implementation of the TAXI milestone, with the intention to make the target take-off time (TTOT) more accurate. When a departing flight receives the TAXI clearance, the SUM re-calculates its TTOT utilizing all other necessary data thus making the TTOT more likely to be attained. An analysis has been carried out to verify if the TTOT actually improves.

\section{Electronic flight strip system}

Each strip, as shown on the following Fig. 1, represents a single flight.

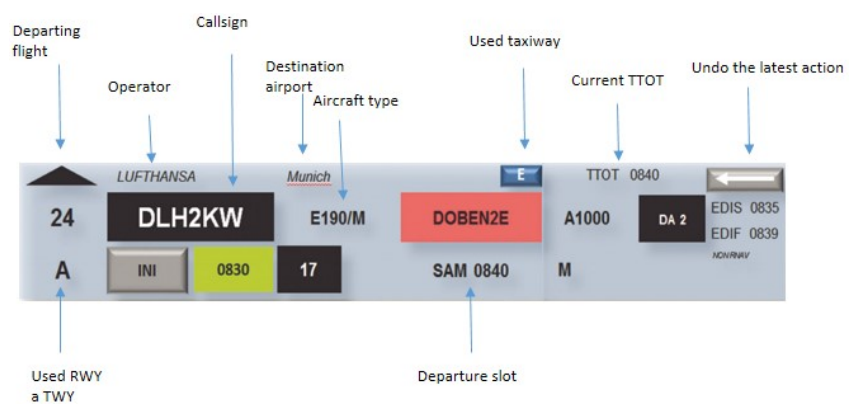

Figure 1. Electronic flight strip.

It contains all necessary information and click-on tiles which are used to issue specific clearances, e.g. TAXI, DEICE, STARTUP, TAKEOFF etc. The EFSS uses the five following working positions:

1. Clearance Delivery Dispatcher (CDD)

2. Ground Executive Controller (GEC)

3. Tower Executive Controller (TEC)

4. Tower Planning Controller (TPC)

5. Tower Supervisor

These positions may be detached or integrated depending on the current traffic intensity. The following diagram (Fig. 2) depicts the tasks and competencies of each position.

\section{Clearance input analysis}

The Air Navigation Services (ANS) of the Czech Republic provided operational data log from two days for the clearance input analysis. The first set of data comes from the 27 th November of 2013 which has seen winter operation including

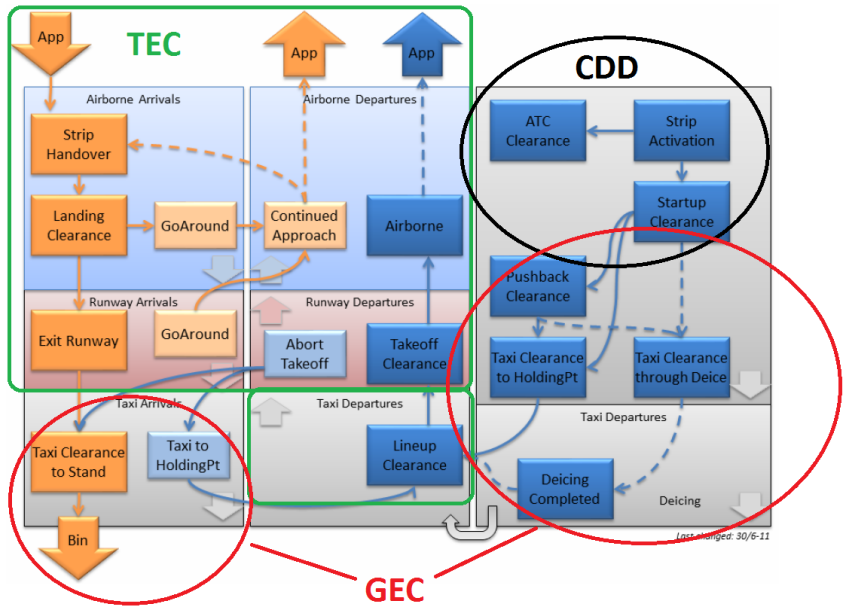

Figure 2. Tasks and competencies of TWR working positions [2].

de-icing. The second set comes from the 20th December of the same year, with heavier traffic but no de-icing. RWY 24 was in use during both analyzed days. Table 1 shows the allocation of working positions to individual workplaces.

Table 1. Allocation of working positions to individual workplaces

\begin{tabular}{lcc} 
Date / Time & $\begin{array}{c}\text { Allocation of } \\
\text { working } \\
\text { positions }\end{array}$ & $\begin{array}{c}\text { Individual } \\
\text { workplaces }\end{array}$ \\
\hline \multirow{2}{*}{20.12 .2013} & CDD & 3 \\
$0: 00$ & GEC & 3 \\
& TEC & 3 \\
& TPC & 3 \\
\hline \multirow{2}{*}{20.12 .2013} & CDD & 1 \\
$5: 56$ & GEC & 2 \\
& TEC & 3 \\
\hline \multirow{2}{*}{20.12 .2013} & TPC & 4 \\
$11: 08$ & CDD & 2 \\
& GEC & 3 \\
& TEC & 4 \\
\hline \multirow{2}{*}{20.12 .2013} & TPC & 3 \\
$21: 00$ & CDD & 3 \\
& GEC & 3 \\
\hline
\end{tabular}

In peak times, each position is operated by a single ATCO as seen from the Tab. 1. In off-peak times, the positions may be merged to a single workplace. That is mostly true for night operations. Regarding issued clearances, the busiest position is TEC. During a morning rush hour, between 9-10 AM on the 20th December, the TEC issued 100 clearances, which average to one clearance every 36 seconds. The second busiest was GEC with 77 clearances, followed by CDD with 32 clearances. TPC does not issue any clearances as it is only a supervisor's position. 


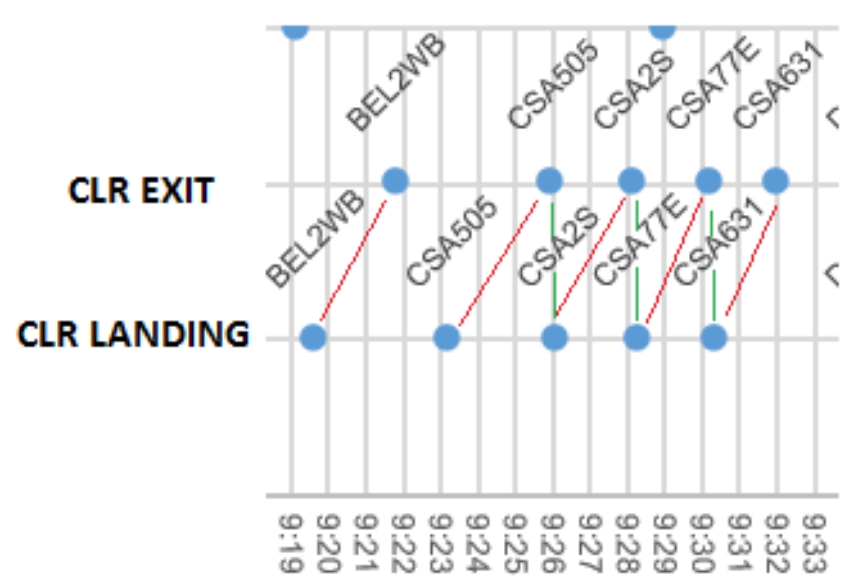

Figure 3. The example of clearances time sequence.

The positions were merged into one at 21:00, which also started that night's busiest hour. The total amount of clearances issued by the merged position between 21:00-22:00 was 60.

In order to find out whether the ATCOs issue certain clearances at the same time or in a close consecutiveness, the authors of this research utilized diagrams, which turned out to be very illustrative. However, as their dimensions are rather large, only a small cut-out section will be presented here to demonstrate their logics.

Blue dots on the diagram shown in Fig. 3, represent the issue of clearances for specific flights. The time of issue can be approximated from the timeline below. The red lines connect the same flights while the green lines highlight clearances issued closely after one another. The pattern is apparent: the ATCO in this time segment repeatedly issued LANDING clearance right after the previous arriving flight received EXIT clearance.

The same method, along with conventional statistics [3], was used repeatedly for all three controlling positions and led to the following conclusions:

- TEC position: LINEUP and TAKEOFF clearances are often issued together, especially in off-peak times.

- GEC position:

- TAXI and PARKING clearances are issued simultaneously for $60 \%$ of analyzed flights.

- ATCOs frequently issue multiple TAXI or PARKING clearances for different aircraft at the same time.

- Clearances are often issued together in clusters which are separated by as much as several minutes. An example is a situation when one aircraft receives PARKING clearance, and another one a TAXI clearance right after that.

- CDD position: ATC and STARTUP clearances are issued together in about $35-50 \%$ of cases, especially in off-peak traffic.

\section{The implementation of TAXI milestone to SUM}

The accuracy of TTOT calculated by SUM was not deemed sufficient, so in July 2014 the ANS implemented the TAXI milestone to improve the ATOT-TTOT accuracy. The function can be described as follows:

- A table of taxi times contains information of taxi duration between every apron and the holding point (HP) of respective RWY.

- A table of pushback times contains information of pushback duration for each apron depending on the aircraft Wake Turbulence Category (WTC).

- Estimated Taxi Time (EXOT) is the sum of pushback time and taxi time for the relevant apron-RWY combination. The sum of TSAT + EXOT is used to calculate TTOT.

- When TAXI clearance is issued by EFSS, SUM can now recalculate TTOT as TAXI + taxi time.

The research begun with one data from one week before and one week after the implementation. The number of TTOT recalculations after AOBT decreased by $11 \%$ from 3.68 to 3.29 per aircraft. However, student's T-test has been performed on the data sample and suggested that this decrease is not significant.

The data sample was then increased to cover a period of two months prior to the implementation and two following months. As shown on the graph of traffic intensity below (Fig. 4), the traffic flow was very consistent, with 11366 departures in the first period and 11319 in the second.

Characteristics for each individual RWY show a significant improvement: a decrease of TTOT recalculations before start-up (STU). The outcomes for the two main RWYs are following:

- RWY 24: An average of 3.1 recalculations per each departing aircraft before the implementation, and 2.7 after.

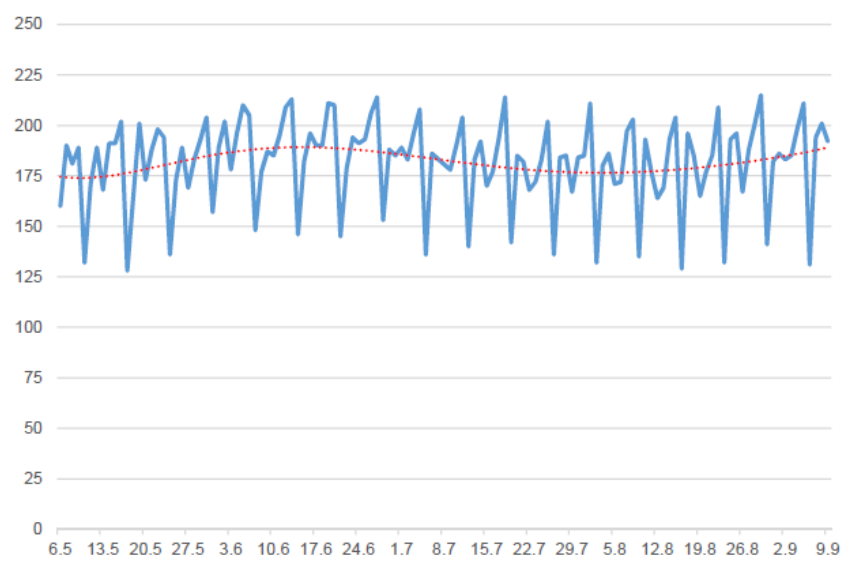

Figure 4. The graph of trafic intensity. 
- RWY 06: An average of 4.2 recalculations per each departing aircraft before the implementation, and 3.2 after.

\section{Accuracy of TTOT in comparison to ATOT}

The next target was to assess any improvements in TTOT accuracy in comparison to ATOT. The dataset remained the same, but this time the analysis focused on the ATOT-TTOT difference. Because the TTOT changes multiple times for most departing flights during the handling process, these changes had to be taken into consideration. Three TTOTs were logged for each departing flight, each of them valid in one of the following stages: after the STARTUP, OFFBLOCK and TAXI clearances were issued. These TTOTs were compared to ATOTs for the respective flights. Results from the two months period before the introduction of the TAXI milestone were compared to the results from the two months period after the introduction to present the following results:

- The accuracy of TTOT valid at the time of STARTUP clearance did not change significantly.

- The accuracy of TTOT valid at the time of PUSHBACK clearance did not change significantly.

- The accuracy of TTOT valid at the time of TAXI clearance improved very considerably.

The results confirmed the success of the TAXI milestone implementation as there was no reason for TTOT improvement in any other stage than after the TAXI clearance has been issued. The analysis results are evident on the Fig. 5.

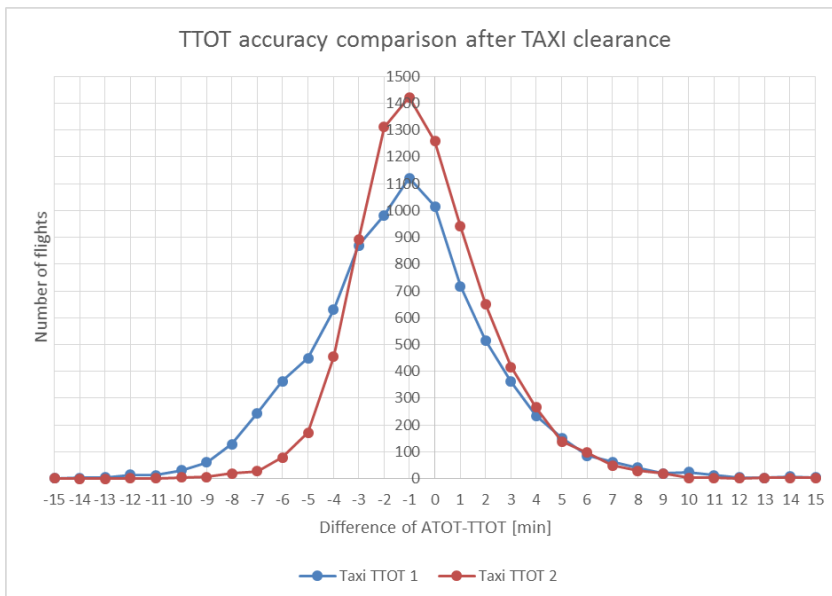

Figure 5. Figure 5. The difference of TTOTs from ATOTs for all flights.

The histogram above shows the difference of TTOTs from ATOTs for all the flights in the two two-month periods. The blue line represents the period before the TAXI milestone was implemented, while the red line represents the later one. The horizontal axis marks the difference of TTOT from ATOT in [min]: negative values represent early departures, while positive values represent minor delays. The mean and modus values for both periods are in -1 minute, but the variance is much lower as the number of departures was almost the same.

\section{TTOT accuracy for RWY 06}

Most of the tests have so far been universal, not taking into account individual RWYs or aprons. That changes with the last analysis, which is taking into consideration both the RWY and each apron that has been used during the observed time period. The results for RWY 06, which will be resented here, show that the average ATOT-TTOT difference in the first time period was $-2.93 \mathrm{~min}$. Or in other words, an average aircraft departing from RWY 06 got airborne 2.93 minutes prior to its TTOT. After the TAXI milestone implementation, that difference improved by almost two minutes to $-1.07 \mathrm{~min}$. Figure 6 shows a portion of a graph that covers all the aprons used more than 10 times in each analyzed time period.

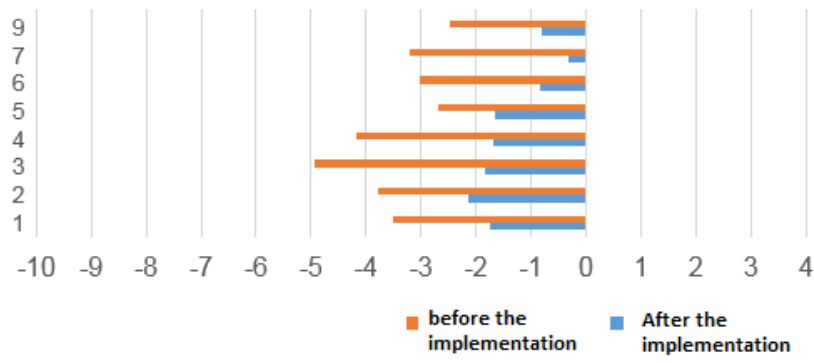

Figure 6. Average difference of TTOT from ATOT for particular aprons.

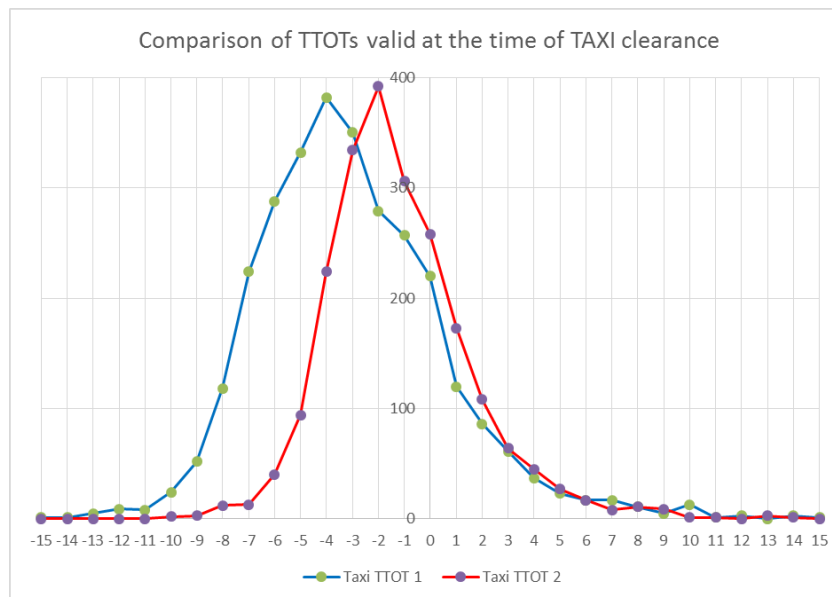

Figure 7. Comparison of difference of TTOT from ATOT before and after TAXI milestone implementation.

The apron number is shown on the vertical axis, while the average difference of TTOT from ATOT is represented by a colored horizontal bar - an orange bar for the first time period and a blue bar for the second. The horizontal axis shows the length of the delay in minutes. The improvement in accuracy is evident and this data can be used to further adjustment of 
taxi times. The overall accuracy for all aprons is shown in Fig. 7.

The blue line shows the difference of TTOT from ATOT for the time period before the TAXI milestone implementation. The red line shows the new data from the period after the implementation. The delay in minutes is shown on the horizontal axis while the number of respective flights can be read from the vertical axis.

The histogram does not represent an equal amount of data as the first time period has seen 2955 departures from RWY 06 while only 2149 flights departed in the second. Nevertheless, the improvement in TTOT accuracy is apparent and is corresponding with the previous graph.

\section{Conclusion}

The EFSS analysis shows that ATCOs often issue either the same or various clearances together, at the same time. That happens for example with consequent clearances concerning a single flight in an off-peak time, when the ATCOs assume that it is safe to do so without having to worry about other traffic. The analysis has proven that TAXI milestone implementation was a success. The following findings confirm the improvements:

- Decrease in TTOT recalculations before and after StartUp.

- Increased ATOT-TTOT accuracy, both overall and in regard to individual aprons and RWYs.

\section{Acknowledgments}

This paper was prepared as output of project work on project Air traffic control in master study program.

\section{References}

[1] Air Navigation Services of the Czech Republic. Annual report 2013, 2014. URL http://www.rlp.cz/ spolecnost/vykonnost/vyrocnizpravy/VZ_ RLP_superfinal_2013.pdf. [Online].

[2] Strip 2014: Zpravodaj Řízení letového provozu České republiky, 15, jan 2014.

[3] D.J. Sheskin. Handbook of Parametric and Nonparametric Statistical Procedures. Chapman \& Hall/CRC, 2003. ISBN 1420036262. 